\title{
A NOTE ON REDUCTIONS OF IDEALS RELATIVE TO AN ARTINIAN MODULE
}

\author{
by A.-J. TAHERIZADEH
}

(Received 3 December, 1991; revised 26 March, 1992)

Introduction. The concept of reduction and integral closure of ideals relative to Artinian modules were introduced in [7]; and we summarize some of the main aspects now.

Let $A$ be a commutative ring (with non-zero identity) and let $\mathfrak{a}, \mathfrak{b}$ be ideals of $A$. Suppose that $M$ is an Artinian module over $A$. We say that $a$ is a reduction of $\mathfrak{b}$ relative to $M$ if $\mathfrak{a} \subseteq \mathfrak{b}$ and there is a positive integer $s$ such that

$$
\left(0:_{M} a \mathfrak{b}^{s}\right)=\left(0:{ }_{M} \mathfrak{b}^{s+1}\right)
$$

An element $x$ of $A$ is said to be integrally dependent on a relative to $M$ if there exists $n \in \mathbb{N}$ (where $\mathbb{N}$ denotes the set of positive integers) such that

$$
\left(0::_{M} \sum_{i=1}^{n} x^{n-i} a^{i}\right) \subseteq\left(0:_{M} x^{n}\right)
$$

It is shown that this is the case if and only if $\mathfrak{a}$ is a reduction of $\mathfrak{a}+A x$ relative to $M$; moreover

$$
\overline{\mathfrak{a}}=\{x \in A: x \text { is integrally dependent on } \mathfrak{a} \text { relative to } M\}
$$

is an ideal of $A$ called the integral closure of $a$ relative to $M$ and is the unique maximal member of

$$
\mathscr{C}=\{\mathfrak{b}: \mathfrak{b} \text { is an ideal of } A \text { which has } \mathfrak{a} \text { as a reduction relative to } M\} .
$$

In [3] the concept of the relevant component of an ideal $I$ (denoted by $I^{*}$ ) of a Noetherian ring $R$ was introduced; moreover the arguments in [3], [5] prove that $I^{*}$ is an interesting and useful ideal.

Now, an interesting question arises: whether there are, in the Artinian situation, some companion results to those discussed for instance in [3].

The purpose of this paper is to show that the Artinian property of the $A$-module $M$ enables us to define and develop a satisfactory concept of the relevant component of an ideal relative to $M$; and the author hopes that this note presents topics for further research.

1. Notation and preliminary results. Throughout the paper $M$ is an Artinian module over the commutative ring $A$ (with non-zero identity) and $\mathfrak{a}$ is an ideal of $A$. We use $\mathbb{N}$ to denote the set of positive integers and $\mathbb{Z}$ to denote the set of integers.

Definition and Remark 1.1. The relevant component of the ideal a of $A$ relative to $M$ is denoted by $\mathfrak{a}^{*}$ and defined as follows:

$$
\mathfrak{a}^{*}=\operatorname{ann}_{A}\left(\bigcap_{i \geqslant 0} \mathrm{a}^{i}\left(0:_{M} a^{i+1}\right)\right) .
$$

It follows from the minimal condition that, for large enough $k, \mathfrak{a}^{*}=\operatorname{ann}_{A}\left(\mathfrak{a}^{k}\left(0:_{M} \mathfrak{a}^{k+1}\right)\right)$,

Glasgow Math. J. 35 (1993) 219-224. 
also we note that $\mathfrak{a} \subseteq \mathfrak{a}^{*}$ and

$$
\mathfrak{a}^{*}=\left(0:_{A} \mathfrak{a}^{k}\left(0:_{M} \mathfrak{a}^{k+1}\right)\right)=\left(\left(0:_{M} \mathfrak{a}^{k}\right):_{A}\left(0:_{M} \mathfrak{a}^{k+1}\right)\right) .
$$

In what follows we shall show that, in fact, when $\mathfrak{a}$ is a co-regular ideal (for definition of a co-regular ideal, see 1.6 below)

$$
\left(0:{ }_{M} a^{* k}\right)=\left(0:{ }_{M} a^{k}\right) \text { for all large enough } k \text {. }
$$

Thus, $\mathfrak{a}$ is a very special kind of reduction of $\mathfrak{a}^{*}$ relative to $M$, since we have, for all large enough $k$,

$$
\left(0:_{M} \mathfrak{a}^{k}\right)=\left(0:_{M} \mathfrak{a}^{* k}\right) \subseteq\left(0:_{M} \mathfrak{a} \mathfrak{a}^{* k-1}\right) \subseteq\left(0:_{M} \mathfrak{a}^{2} \mathfrak{a}^{* k-2}\right) \subseteq \ldots \subseteq\left(0:_{M} \mathfrak{a}^{k}\right)
$$

Hence, for all large enough $k$,

$$
\left(0:{ }_{M} a^{* k}\right)=\left(0:_{M} a a^{* k-1}\right)=\ldots=\left(0:_{M} a^{k-1} a^{*}\right)=\left(0:_{M} a^{k}\right) .
$$

For the main result, we need a few preliminary lemmas which will be given below. all $n \geqslant r$,

Lemma 1.2. Suppose $x \in A$ is such that $x M=M$. Then there exists $r \in \mathbb{N}$ such that, for

$$
x\left(0:_{M} \mathfrak{a}^{n}\right) \supseteq\left(0:_{M} \mathfrak{a}^{n-r}\right) .
$$

Proof. By the Artin-Rees lemma for Artinian modules [1, Proposition 3], there is $r \in \mathbb{N}$ such that, for all $n \geqslant r$,

$$
\left(0:_{M} x\right)+\left(0:_{M} \mathfrak{a}^{n}\right)=\left(\left(\left(0:_{M} x\right)+\left(0:_{M} \mathfrak{a}^{r}\right)\right): \mathfrak{a}^{n-r}\right) \supseteq\left(\left(0:_{M} x\right): \mathfrak{a}^{n-r}\right) .
$$

On the other hand, we have, for all $n \in \mathbb{N}$,

$$
\left(0:_{M} x\right)+\left(0:_{M} \mathfrak{a}^{n}\right)=\left(x\left(0:_{M} \mathfrak{a}^{n}\right): A x\right) .
$$

So it follows from (2) and (3) that, for all $n \geqslant r$,

$$
\left(x\left(0:_{M} \mathfrak{a}^{n}\right): A x\right) \supseteq\left(\left(0:_{M} x\right): \mathfrak{a}^{n-r}\right) .
$$

Now, let $y \in\left(0: M a^{n-r}\right)$, where $n \geqslant r$. Then, since $x M=M$, there is $m \in M$ such that $y=x m$. Hence $m \in\left(\left(0:_{M} x\right): \mathfrak{a}^{n-r}\right)$. Thus $m \in\left(x\left(0:_{M} \mathfrak{a}^{n}\right): A x\right)$ by (4). Therefore $y=x m \in x\left(0:{ }_{M} \mathfrak{a}^{n}\right)$. The proof is now complete.

For the main result (Theorem 2.2), we need the concept of a superficial element relative to a module, which we introduced and developed in [8, Chapter III, \$7]. However, for readers' convenience, we recall the main points of the theory.

Let $N$ be an $A$-module and $\mathfrak{b}$ a proper ideal of $A$. Then $x \in \mathfrak{b}^{s}(s \in \mathbb{N})$ is called a superficial element of order $s$ for $b$ relative to $N$ if there is $c \in \mathbb{N}$ such that for all $n>c$

$$
\left(0:_{N} \mathfrak{b}^{n}\right)=x\left(0:_{N} \mathfrak{b}^{n+s}\right)+\left(0:_{N} \mathfrak{b}^{c}\right) .
$$

For the next two propositions, suppose that $\mathfrak{a}=\left(a_{1}, \ldots, a_{k}\right)$ is a finitely generated ideal of $A$ and $M$ an Artinian $A$-module and $T$ an indeterminate. Let $R=$ $A\left[a_{1} T, \ldots, a_{k} T\right]$ be the (small) Rees ring of $A$ with respect to $a$ and graded in the usual way by $\mathbb{Z}$. Set $G=\bigoplus_{n \in \mathbb{Z}} G_{n}$, where, for $n \in \mathbb{Z}$,

$$
G_{n}=\left\{\begin{array}{lll}
0 & \text { if } & n>0 \\
\left(0:_{M} \mathfrak{a}^{-n+1}\right) /\left(0:_{M} a^{-n}\right) & \text { if } & n \leqslant 0 .
\end{array}\right.
$$


Let $x_{1}, \ldots, x_{k}$ be indeterminates over $A$ and let $R^{\prime}=\bigoplus_{n \in \mathbb{Z}} R_{n}^{\prime}$ denote the ring $A\left[x_{1}, \ldots, x_{k}\right]$ graded in the usual way, so that $R_{n}^{\prime}=0$ for $n<0$. Now turn $G$ into a graded $R^{\prime}$-module using the ideas of Kirby in [1, p. 54]:if $n$ is an integer with $n<0$, $m \in\left(0:_{M} \mathfrak{a}^{-n+1}\right)$ and $1 \leqslant i \leqslant k$, put

$$
x_{i}\left(m+\left(0:_{M} \mathfrak{a}^{-n}\right)\right)=a_{i} m+\left(0:_{M} a^{-n-1}\right) \in G_{n+1} .
$$

Next there exists a surjective ring homomorphism $\varphi: R^{\prime} \rightarrow R$ such that $x_{i}(1 \leqslant i \leqslant k)$ is mapped into $a_{i} T$ and $\operatorname{Ker} \varphi \subseteq \operatorname{Ann}_{R^{\prime}} G$. Thus $G$ has a structure as an $R$-module. Now we easily deduce the following proposition. (For a proof, see [8, p. 82, 7.3].)

Proposition 1.3. Let the notation and assumptions be the same as above. Then $x \in \mathfrak{a}^{s}$ $(s \in \mathbb{N})$ is a superficial element of order $s$ for a relative to $M$ if and only if there is $c^{\prime} \in \mathbb{N}$ such that, for all $n>c^{\prime}$

$$
\left(x T^{s}\right) G_{-n-s}=G_{-n} .
$$

The next proposition shows that whenever $M$ is an Artinian $A$-module, superficial elements do exist.

Proposition 1.4 [8, p. 83, 7.4]. Let $M$ be an Artinian A-module and a a proper ideal of $A$. Then there exists an element $x$ of $A$ such that $x$ is a superficial element of order $s$ for $a$ relative to $M$.

Proof. By [1, Lemma 3], we can (and do) assume that $\mathfrak{a}$ is finitely generated. Suppose the notation is as in Proposition 1.3 and, further, $\mathfrak{b}$ denotes the ideal $\sum_{i=1}^{k} R\left(a_{i} T\right)$ of $R$. Then, by [9, Lemma 2.2], $G$ is an Artinian $R$-module and hence graded Artinian (that is, satisfies the minimal condition for homogeneous submodules). By [6, Proposition 2.4], $G$ has a reduced graded-secondary representation,

$$
G=N_{1}+\ldots+N_{r^{\prime}}+N_{r^{\prime}+1}+\ldots+N_{t},
$$

where each $N_{i}$ is a graded secondary homogeneous submodule of $G$ and $\sqrt{\left(0: N_{i}\right)}$ is a homogeneous prime ideal $\mathfrak{q}_{i}$, say, of $R(1 \leqslant i \leqslant t)$. Further suppose that the $N_{i}$ are numbered so that

$$
\begin{gathered}
\mathfrak{b} \nsubseteq \mathfrak{q}_{i} \text { for } i=1, \ldots, r^{\prime}, \\
\mathfrak{b} \subseteq \mathfrak{q}_{i} \text { for } i=r^{\prime}+1, \ldots, t .
\end{gathered}
$$

Then, by the same argument as in [6, Theorem 3.1], we find $c \in \mathbb{N}$ and $f_{s} \in R_{s}$ homogeneous of degree $s(s \in \mathbb{N})$, such that $f_{s} \notin \bigcup_{i=1}^{r^{\prime}} \mathfrak{q}_{i}$ and $f_{s} G_{-m-s}=G_{-m}$ for all $m>c$.

Suppose that $f_{s}=x T^{s}$ for some $x \in \mathfrak{a}^{s}$. Then

$$
\left(x T^{s}\right) G_{-m-s}=G_{-m} \text { for all } m>c,
$$

and the result follows from Proposition 1.3.

Lemma 1.5. Let $M$ be an Artinian A-module and a an ideal of $A$. Then the following are equivalent:

(i) $M=\mathfrak{a} M$,

(ii) $M=x M$ for some $x \in a$. 
Proof. We adapt the proof of $[2,2.8]$. Let $M=N_{1}+\ldots+N_{r}$ be a reduced secondary representation with $\sqrt{\left(0: N_{i}\right)}=\mathfrak{p}_{i}, 1 \leqslant i \leqslant r$.

(i) $\Rightarrow$ (ii). Suppose $a \subseteq \mathfrak{p}_{i}$ for some $1 \leqslant i \leqslant r$. Then, by [1, Lemma 3], there is a finitely generated ideal $\mathfrak{b}$ such that $\mathfrak{b} \subseteq \mathfrak{a}$ and $\left(0:_{M} \mathfrak{b}^{n}\right)=\left(0:_{M} \mathfrak{a}^{n}\right)$ for all $n \in \mathbb{N}$. Now for each $x \in \mathfrak{b}$ there is $t_{x} \in \mathbb{N}$ such that $x^{t_{x}} N_{i}=0$. Thus, since $\mathfrak{b}$ is finitely generated, there is $\iota \in \mathbb{N}$ such that $\mathfrak{b}^{t} N_{i}=0$, and so $N_{i} \subseteq\left(0:_{M} \mathfrak{b}^{t}\right)=\left(0:_{M} \mathfrak{a}^{t}\right)$. Hence $\mathfrak{a}^{t} N_{i}=0$. So

$$
M=\mathfrak{a}^{t} M=\sum_{j \neq i} \mathfrak{a}^{t} N_{j} \subseteq \sum_{j \neq i} N_{j} \neq M,
$$

a contradiction. Thus $a \nsubseteq \mathfrak{p}_{i}$ for $1 \leqslant i \leqslant r$, and so $a \nsubseteq \bigcup_{i=1}^{r} \mathfrak{p}_{i}$.

Let $x \in \mathfrak{a} \backslash \bigcup_{i=1}^{r} \mathfrak{p}_{i}$. Then, by $[2,2.6]$, the endomorphism of $M$ given by multiplication by $x$ is surjective, i.e. $M=x M$. Since (ii) $\Rightarrow$ (i) is obvious, the proof of the lemma is now complete.

REMARK. The referee kindly pointed out to me that this lemma (with essentially the same proof) appears as Proposition 3.4 in a paper of Ooishi [5]; and he attributes the result to Matlis.

Definition 1.6. The ideal a of $A$ is called co-regular if $a M=M$. By Lemma 1.5 , this is the case if and only if $x M=M$ for some $x \in \mathfrak{a}$. The element $x$ then is called a co-regular element.

2. Main result. Throughout this section $\mathfrak{a}$ is a co-regular ideal of $A$.

For the main result we need another lemma which is given below.

Lemma 2.1. Let $M$ be an Artinian A-module and a a proper ideal of $A$. Then there exists a superficial element of order $s$ for a relative to $M$ which is co-regular as well.

Proof. With the same notation as in Propositions 1.3 and 1.4 , we note that $\mathfrak{a} \subseteq \mathfrak{p}_{l} \Leftrightarrow \mathfrak{b}=\sum_{j=1}^{k} R\left(a_{j} T\right) \subseteq \mathfrak{B}_{l}(1 \leqslant l \leqslant r)$, where $\mathfrak{B}_{l}=\bigoplus_{n \in \mathbb{Z}}\left(\mathfrak{p}_{l}^{\prime}\right)_{n}$ are primes and are such that

$$
\left(\mathfrak{p}_{l}^{\prime}\right)_{n}=\left\{\begin{array}{lll}
0 & \text { if } & n<0, \\
\left(\mathfrak{p}_{l} \cap \mathfrak{a}^{n}\right) T^{n} & \text { if } & n \geqslant 0
\end{array}\right.
$$

(with the convention that $\mathfrak{a}^{n}=A$ for $n \leqslant 0$ ). Now, by the arguments used in Propositions 1.3 and 1.4 and the identity above,

$$
\begin{array}{lc}
\mathfrak{b} \nsubseteq \mathfrak{q}_{i} & 1 \leqslant i \leqslant r^{\prime}, \\
\mathfrak{b} \nsubseteq \mathfrak{P}_{i} & 1 \leqslant i \leqslant r .
\end{array}
$$

So, by $[9$, Lemma 2.1$]$, there is $h_{0} \in \mathbb{Z}$ such that, for all $h \geqslant h_{0}, R_{h} \nsubseteq\left(\bigcup_{i=1}^{r} \mathfrak{B}_{i}\right) \cup\left(\bigcup_{i=1}^{r^{\prime}} \mathfrak{q}_{i}\right)$. Let $s \in \mathbb{N}$ be such that $s \geqslant h_{0}$ and $x T^{s}=f_{s} \in R_{s} \backslash\left(\bigcup_{i=1}^{r} \mathfrak{B}_{i}\right) \cup\left(\bigcup_{i=1}^{r^{\prime}} q_{i}\right)$ for some $x \in a^{s}$. Then, by Propositions 1.3 and 1.4 and (5), $x$ is a superficial element of order $s$ relative to $M$, which is a co-regular element as well. 
TheOREM 2.2. Let $M$ be an Artinian A-module and a a co-regular ideal of $A$. Then $\left(0:_{M} a^{* k}\right)=\left(0:_{M} a^{k}\right)$, for all large $k$. Further, $a^{*}$ is the largest ideal with this property.

Proof. By Lemma 2.1, there exists a positive integer $s$ and an element $x \in a^{s}$ such that $x$ is a superficial element of $A$ of order $s$ relative to $M$ which is a co-regular element. Thus there is $c \in \mathbb{N}$ such that, for all large enough $n$,

$$
\left(0:{ }_{M} \mathfrak{a}^{n}\right)=x\left(0:_{M} \mathfrak{a}^{n+s}\right)+\left(0:_{M} \mathfrak{a}^{c}\right) .
$$

Now it follows from Lemma 1.2 and (6) that for all large enough $n$, say $n \geqslant t$,

$$
x\left(0:_{M} \mathfrak{a}^{n+s}\right)=\left(0:_{M} \mathfrak{a}^{n}\right) .
$$

Let $m=t+s$. Then, by (7), we have, for all $j \geqslant 0$,

$$
\begin{aligned}
\left(0:_{M} \mathfrak{a}^{m+j}\right)=x\left(0:_{M} \mathfrak{a}^{m+j+s}\right) & \subseteq \mathfrak{a}^{s}\left(0:_{M} \mathfrak{a}^{m+j+s}\right) \\
& \subseteq \mathfrak{a}^{s-1}\left(0:_{M} \mathfrak{a}^{m+j+s-1}\right) \\
& \subseteq \cdots \subseteq \mathfrak{a}\left(0:_{M} \mathfrak{a}^{m+j+1}\right) \\
& \subseteq\left(0:_{M} \mathfrak{a}^{m+j}\right) .
\end{aligned}
$$

Thus $\mathfrak{a}\left(0:_{M} \mathfrak{a}^{l+1}\right)=\left(0:_{M} \mathfrak{a}^{\prime}\right)$ for all $l \geqslant m$. Therefore

$$
\mathfrak{a}^{i}\left(0:{ }_{M} \mathfrak{a}^{l+i}\right)=\left(0:_{M} \mathfrak{a}^{l}\right) \text { for all } l \geqslant m, \quad i \geqslant 1 .
$$

Now it follows from the definition of $a^{*}$ that, for large $n$,

$$
\begin{aligned}
\left(0:_{M} \mathfrak{a}^{2}\right) \supseteq\left(0:_{M} \mathfrak{a}^{* 2}\right) & =\left(\left(0:_{M} \mathfrak{a}^{*}\right): \mathfrak{a}^{*}\right) \supseteq\left(\mathfrak{a}^{n}\left(0:_{M} \mathfrak{a}^{n+1}\right):_{A} \mathfrak{a}^{*}\right) \\
& \supseteq \mathfrak{a}^{n}\left(\left(0:_{M} \mathfrak{a}^{n+1}\right):_{A} \mathfrak{a}^{*}\right) \\
& =\mathfrak{a}^{n}\left(\left(0:_{M} \mathfrak{a}^{*}\right):_{A} \mathfrak{a}^{n+1}\right) \\
& \supseteq \mathfrak{a}^{n}\left(\mathfrak{a}^{n}\left(0:_{M} \mathfrak{a}^{n+1}\right):_{A} \mathfrak{a}^{n+1}\right) \\
& \supseteq \mathfrak{a}^{2 n}\left(\left(0:_{M} \mathfrak{a}^{n+1}\right):_{A} \mathfrak{a}^{n+1}\right) \\
& \supseteq \mathfrak{a}^{2 n}\left(0:_{M} \mathfrak{a}^{2 n+2}\right) .
\end{aligned}
$$

Then, we deduce from this (by induction) and (8) that, for large $k,\left(0:_{M} a^{k}\right) \supseteq$ $\left(0:_{M} a^{* k}\right) \supseteq a^{k n}\left(0:_{M} a^{k n+k}\right)=\left(0:_{M} a^{k}\right)$. Therefore $\left(0:_{M} a^{k}\right)=\left(0:_{M} a^{* k}\right)$ for all large $k$.

For the last part, suppose that $\mathfrak{b}$ is a proper ideal of $A$ such that $\left(0:_{M} \mathfrak{b}^{k}\right)=\left(0:_{M} \mathfrak{a}^{k}\right)$ for all large $k$, say, $k \geq t$. Let $l \geqslant 2 t-1$. Then

$$
\begin{aligned}
\left(0:_{M}(\mathfrak{a}+\mathfrak{b})^{l}\right) & =\left(0:_{M} \sum_{i=0}^{l} \mathfrak{a}^{i} \mathfrak{b}^{l-i}\right)=\bigcap_{i=0}^{l}\left(0:_{M} \mathfrak{a}^{i} \mathfrak{b}^{l-i}\right) \\
& =\left(\bigcap_{i=0}^{t-1}\left(\left(0:_{M} \mathfrak{b}^{l-i}\right): \mathfrak{a}^{i}\right)\right) \cap\left(\bigcap_{i=t}^{l}\left(\left(0:_{M} \mathfrak{a}^{i}\right): \mathfrak{b}^{l-i}\right)\right) .
\end{aligned}
$$

Now by our assumption if $0 \leqslant i \leqslant t-1$, then $\left(0:_{M} \mathfrak{b}^{l-i}\right)=\left(0:{ }_{M} \mathfrak{a}^{l-i}\right)$ and for $t \leqslant i \leqslant l$, $\left(0:_{M} \mathfrak{a}^{i}\right)=\left(0:_{M} \mathfrak{b}^{i}\right)$. Thus

$$
\begin{aligned}
\left(0:_{M}(\mathfrak{a}+\mathfrak{b})^{l}\right) & =\left(\bigcap_{i=0}^{t-1}\left(\left(0:_{M} \mathfrak{a}^{l-i}\right): \mathfrak{a}^{i}\right)\right) \cap\left(\bigcap_{i=t}^{l}\left(\left(0:_{M} \mathfrak{b}^{i}\right): \mathfrak{b}^{l-i}\right)\right) \\
& =\left(0:_{M} \mathfrak{a}^{l}\right) \cap\left(0:_{M} \mathfrak{b}^{l}\right)=\left(0:_{M} \mathfrak{a}^{l}\right)=\left(0:_{M} \mathfrak{b}^{l}\right) .
\end{aligned}
$$


Therefore, if $x \in b$ and $k$ is large enough, then

$$
\left(0:_{M}(\mathfrak{a}+\mathfrak{b})^{l}\right) \subseteq\left(0:_{M} \sum_{i=0}^{l} x^{l-i} \mathfrak{a}^{i}\right) \subseteq\left(0:_{M} \mathfrak{a}^{l}\right)
$$

So $\left(0:_{M} \sum_{i=0}^{l} x^{l-i} \mathfrak{a}^{i}\right)=\left(0:_{M} \mathfrak{a}^{\prime}\right)$ and hence $x\left(0:{ }_{M} \mathfrak{a}^{\prime}\right) \subseteq\left(0:_{M} \mathfrak{a}^{\prime-1}\right)$. Now the result follows from identity (1).

Corollary 2.3. Let $a, a^{*}$ be the same as in 2.2 and $\mathfrak{b}$ an ideal of $A$ such that $\mathfrak{a} \subseteq \mathfrak{b} \subseteq \mathfrak{a}^{*}$. Then $\mathfrak{b}^{*}=\mathfrak{a}^{*}$ and in particular, $\mathfrak{a}^{* *}=\mathfrak{a}^{*}$.

Proof. Since $\mathfrak{a} \subseteq \mathfrak{b} \subseteq \mathfrak{a}^{*}$, we have,

$$
\left(0:{ }_{M} \mathfrak{a}^{* k}\right) \supseteq\left(0:_{M} \mathfrak{b}^{k}\right) \supseteq\left(0:_{M} \mathfrak{a}^{k}\right) \text { for all } k \geqslant 0 .
$$

Hence, it follows from this and Theorem 2.2 that

$$
\left(0: \mathfrak{l}^{k}\right)=\left(0:_{M} \mathfrak{a}^{k}\right) \quad \text { for all large } k .
$$

Now, using (1) and (9), we deduce that $\mathfrak{b}^{*}=\mathfrak{a}^{*}$. Also

$$
\left(0:{ }_{M} \mathfrak{b}^{k}\right)=\left(0:_{M} \mathfrak{a}^{* k}\right) \text { for all large } k \text {. }
$$

Using identity (1) again, we see at once that $\mathfrak{a}^{* *}=\mathfrak{b}^{*}\left(=\mathfrak{a}^{*}\right)$.

ACKNOWLEDGMENT. I should like to thank the referee for his useful suggestions, and the Arak Teacher Training University for their financial support.

\section{REFERENCES}

1. D. Kirby, Artinian modules and Hilbert polynomials, Quart. J. Math. Oxford Ser. (2) 24 (1973), 47-57.

2. I. G. Macdonald, Secondary representation of modules over a commutative ring, Sympos. Math. 11 (1973), 23-43.

3. A. Mirbagheri and L. J. Ratliff Jr, On the relevant transform and the relevant component of an ideal, J. Algebra 111 (1987), 507-519.

4. A. Ooishi, Matlis duality and the width of a module, Hiroshima Math. J. 6 (1976), 573-587.

5. L. J. Ratliff Jr and David E. Rush, Two notes on reduction of ideals, Indiana Univ. Math. J. 27 (1978), 929-934.

6. R. Y. Sharp, Asymptotic behaviour of certain sets of attached prime ideals, J. London Math. Soc. (2) 34 (1986), 212-218.

7. R. Y. Sharp and A.-J. Taherizadeh, Reductions and integral closures of ideals relative to an Artinian module, J. London Math. Soc. (2) 37 (1988), 203-218.

8. A.-J. Taherizadeh, Behaviour of ideals relative to Artinian modules over commutative rings (Ph.D. thesis, University of Sheffield, 1987).

9. A.-J. Taherizadeh, On Asymptotic values of certain sets of attached prime ideals, Glasgow Math. J. 30 (1988), 293-300.

Department of Mathematics

Teacher Training University

49 Mofateh Ave

Tehran, Iran
Current Address:

Department of Mathematics

Arak Teacher Training University Beheshti Ave

ARAK, Iran 